\title{
Impact of Tumor Size as a Prognostic Factor After One Stage Liver Resection for Solitary Hepatocellular Carcinoma in Cirrhotic Patients
}

\author{
Alhassan Mohamed Hassan", Amir Fawzy Abdelhamid, Hosam Barakat Barakat, \\ Soliman Mohamed Soliman ${ }^{1}$, Hossamaldin Mohamed Soliman', Mohamed Abdalla Hablus ${ }^{1}$, \\ Mahmoud Mostafa Alshareef ${ }^{1}$ \\ ${ }^{1}$ Department of Surgery, Faculty of Medicine, Tanat University, Tanta, Egypt \\ ${ }^{2}$ Departement of Surgery, Menofeya National Liver Insistute, Menofeya University, Shebin El-kom, Egypt
}

Email address:

Dr.alhassan94@gmail.com (A. M. Hassan), Hosam123@yahoo.com (H. B. Barakat)

\section{To cite this article:}

Alhassan Mohamed Hassan, Amir Fawzy Abdelhamid, Hosam Barakat Barakat, Soliman Mohamed Soliman, Hossamaldin Mohamed Soliman, Mohamed Abdalla Hablus, Mahmoud Mostafa Alshareef. Impact of Tumor Size as a Prognostic Factor After One Stage Liver Resection for Solitary Hepatocellular Carcinoma in Cirrhotic Patients. Journal of Surgery. Vol. 8, No. 4, 2020, pp. 123-131. doi: $10.11648 /$ j.js.20200804.15

Received: May 27, 2020; Accepted: June 28, 2020; Published: July 7, 2020

\begin{abstract}
Aim: This study is to assess tumor size as a prognostic factor predicting outcomes after one staged hepatectomy for cirrhotic patients with solitary hepatocellular carcinoma. Patients and methods: The study included 41 patients with single hepatocellular carcinoma (HCC) of different sizes who underwent single-stage hepatectomy. Patients were divided according to their tumor size into 2 groups, group A involved patient with tumors $\leq 5 \mathrm{~cm}$ and group B which involved patients with tumors $>5 \mathrm{~cm}$. The effect of the tumor size on overall survival and disease-free survival was studied in both groups. Results: The mean age of the studied groups was $59.60 \pm 6.89$ years. Hepatitis C infection was found $82.9 \%$ of patients. Six patients (17.1\%) received treatment of HCV. All patients were of Child-Pugh class A (77.1\% were scores 5 and $22.9 \%$ were score 6$)$. The median Alpha-fetoprotein (AFP) level was $240 \mathrm{ng} / \mathrm{ml}$. The mean operative time was 186.4 \pm 52.4 min. During the followup period (12-24 months), 12 patients (34.3\%) developed recurrence. The mean time of recurrence was $15.50 \pm 4.23$ months. Cumulative disease-free survival (DFS) at the end of the study was $65.7 \%$. The cumulative overall survival (OAS) proportion at the end of the study was $74.3 \%$. Conclusion: Our results suggest that surgical resection for large HCC is safe and effective and that the first-line treatment for large HCC to be considered is surgical resection in selected patients. Our study showed that hepatectomy for large HCC could be performed with an acceptable morbidity and mortality rate. With the improvement in patient selection and treatment strategy, solitary large HCC is not a contraindication to surgical therapy.
\end{abstract}

Keywords: Tumor Size, Solitary HCC, Resection, Single Stage

\section{Introduction}

Hepatocellular carcinoma (HCC) is the most common primary tumor of the liver representing approximately $80 \%$ - $90 \%$ of primary hepatic malignancies and it represents the $3^{\text {rd }}$ leading cause of neoplasm related deaths worldwide. HCC is usually associated with cirrhosis whose major cause is viral hepatitis [1]. The treatment of HCC on the cirrhotic basis is complex, the aim to be oncologically radical has always to be balanced with the necessity to minimize organ damage to prevent postoperative liver failure [2, 3]. Liver resection is the mainstay treatment for the majority of HCCs due to organ shortage [4]. With an improved preoperative selection of patients and improvement in surgical techniques and perioperative care, morbidity and mortality of hepatectomy have decreased greatly [5]. Barcelona Clinic Liver Cancer (BCLC) has been approved as guidance for HCC treatment algorithms by the European Association for the Study of Liver (EASL) and the American Association for the Study of Liver Disease (AASLD), BCLC algorithm suggests that resection only in small lesions $(<5 \mathrm{~cm})$, on the contrary, several authors, recently 
reported that liver resection can offer good short and long term results even in patients with $\mathrm{HCC}>5 \mathrm{~cm}[6]$. From this point of view, it is that in literature there are many studies reporting cases of liver resection for $\mathrm{HCC}>8-10 \mathrm{~cm}$ with good results [7]. It is imperative to achieve accurate determination of the liver volume, especially in patients with chronic liver disease or cirrhosis where the size of the remnant liver becomes even more important as a prognostic factor [8]. The quest to reduce intra-hepatic recurrence is disappointing, none of the current neoadjuvant or adjuvants for HCC proved beneficial. [9]. Aim of study is to assess tumor size as a prognostic factor predicting outcomes after one staged hepatectomy for cirrhotic patients with solitary hepatocellular carcinoma.

\section{Patients and Methods}

The study was approved by the Ethics committee of the Faculty of Medicine Tanta University. Any unexpected risks appeared during study was clear to participants and the ethical committee on time. There were adequate provisions to maintain the privacy of the participants and the confidentiality of the data. The present study is a multicentric prospective study which was carried out in the period between February 2017 and February 2019. The aim of our study was to assess the impact of HCC tumor size, as a aprognostic factor, on patients outcomes after one stage hepatectomy. This study included 41 cirrhotic patients who had single HCC of different sizes who underwent single stage hepatectomy at both General Surgery Department, Gastrointestinal, Laparoscopic, and Liver surgery Unit, Tanta University Hospital and National Liver Institute Menofeya University. Patients included were: BCLC stage (0- A-B), Child-Pugh A liver disease, Cirrhotic patients with solitary HCC which is defined as any single lesion of any size without satellite nodules and/or macrovascular invasion at the time of preoperative assessment, Technically respectable tumors, A remnant liver volume of at least $40 \%$ was suitable for $\mathrm{HCC} \geq 5 \mathrm{~cm}$ requiring formal anatomical hepatectomy.

The pre-operative investigations included blood chemistry, serum albumin, serum total bilirubin, hepatitis B \& C markers, alpha-fetoprotein (AFP), abdominal ultrasonography (US), computed tomography (CT) and/or abdominal MRI. All HCC patients were discussed in the multidisciplinary HCC board at Tanta University Hospital. All selected HCC patients for liver resection was ChildTurcutt-Pugh (CTP) class A with no manifestations of clinically significant portal hypertension (including platelet count of less than 100000, splenomegaly and esophageal varices). Additionally, all selected patients were free of any extrahepatic metastasis. Patients were divided according to their tumor size into 2 groups, group A involved patients with tumors $\leq 5 \mathrm{~cm}$ and group $\mathrm{B}$ which involved patients with tumors $>5 \mathrm{~cm}$.

In our study, $\mathrm{J}$ shaped (reverse L) or midline incisions for Lt. Sided lesions were used. A self-retaining liver retractor
(Figure 1) is adopted to provide adequate traction by elevating the costal margin, abdominal exploration was done to exclude the dissemination of malignancy. Intra-operative U/S was done in all cases (Figures 2, 4). Resection was either anatomical or non-anatomical according to tumor size and location (Figures 3, 5, 6, 7).

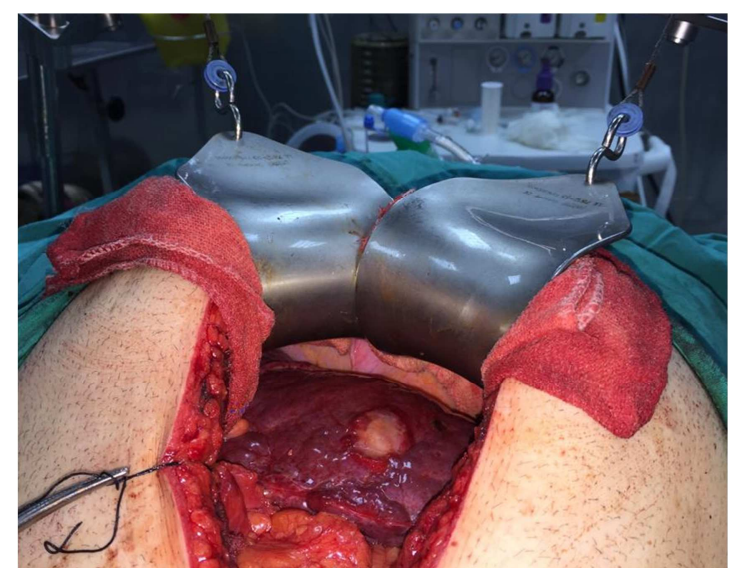

Figure 1. Self-retaining liver retractor.

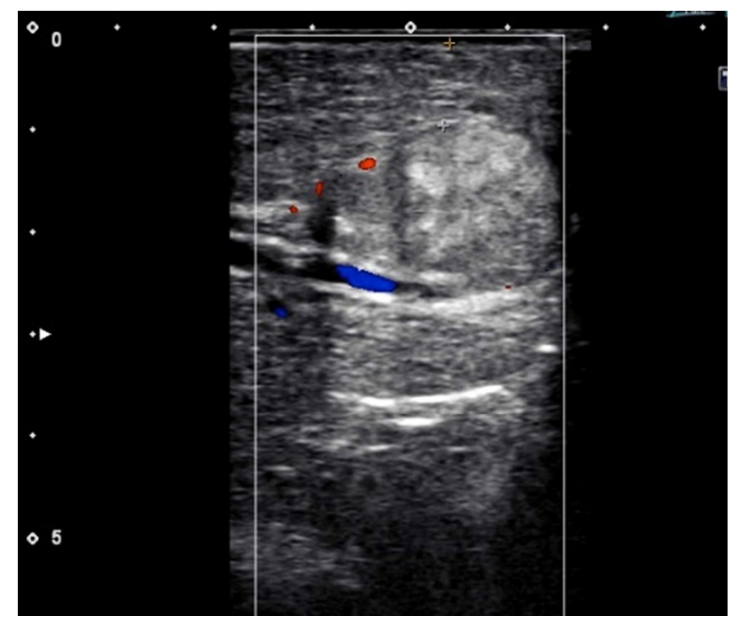

Figure 2. Intraoperative $U / S$.

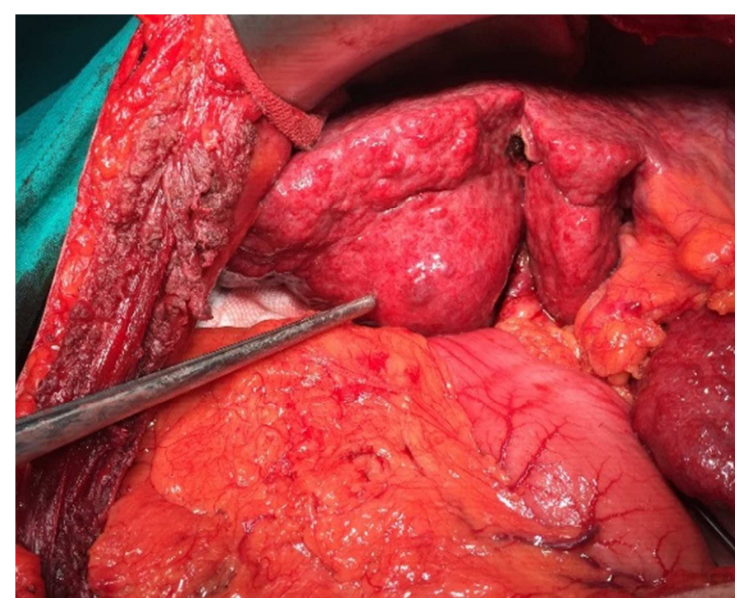

Figure 3. Intraoperative image of seg 6 mass. 


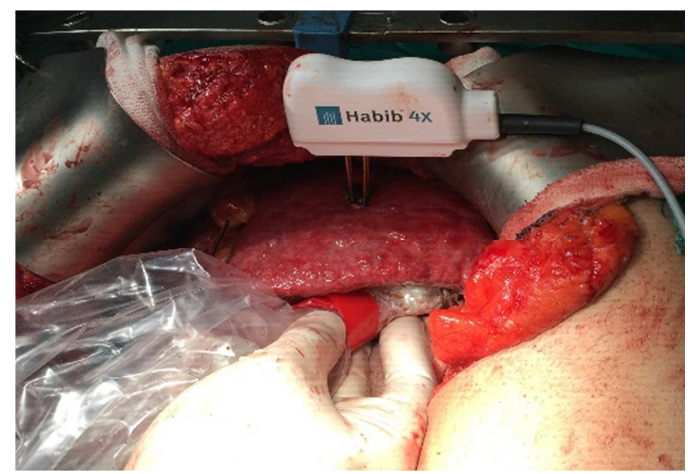

Figure 4. Intraoperative ablation by Habib needle.

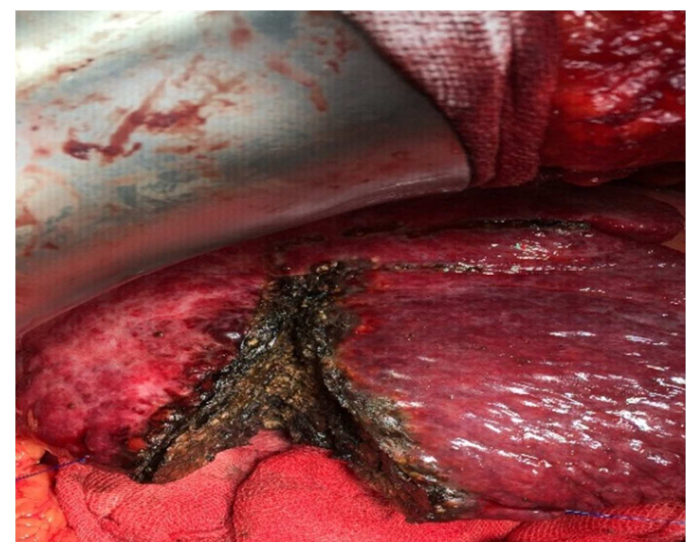

Figure 5. Resection line of the tumor in non-anatomical resection

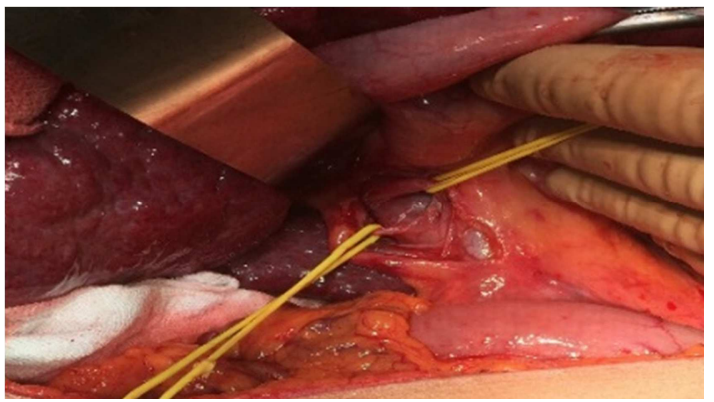

Figure 6. Identification of Rt. hepatic artery and CHD in RT. hepatectomy.

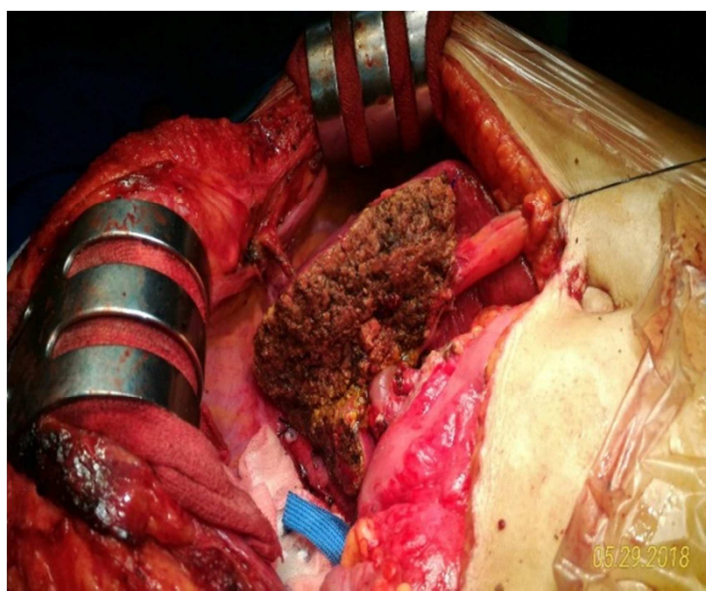

Figure 7. Cut surface of the liver after RT. hepatectomy.

Some patients were admitted to ICU for close observation, other patients were shifted directly to the general ward, the pain was controlled with proper analgesic, Broadspectrum antibiotics were given, Patients were ambulated as early as possible, Drains removed on the $4^{\text {th }}$ or $5^{\text {th }}$ postoperative day unless bile drainage or serosanguinous fluid or ascites $>500$ cc was noted. All specimens retrieved after resections were reviewed by a pathologist. Tumor characteristics included: The number and size of lesions; the degree of differentiation, tumor involvement of the resection margin, the presence of macroscopic and/or microscopic vascular invasion, and the presence of satellite nodules. The severity of postoperative complications during hospital stay was assessed by Clavien Dindo classification (0-5) (Dindo et al, 2004).

Follow up: The primary endpoint was patient mortality and the secondary endpoint was a recurrence. Regarding the time of recurrence, early recurrence was defined as recurrence within 2 years after the initial liver resection. Once a diagnosis of recurrence was confirmed, treatment strategies were proposed based on the decision of a multidisciplinary team.

\subsection{Statistical Analysis of the Data}

Data were fed to the computer and analyzed using IBM SPSS software package version 20.0. (Armonk, NY: IBM Corp) Qualitative data were described using the number and percent. The Kolmogorov-Smirnov test was used to verify the normality of distribution Quantitative data were described using range (minimum and maximum), mean, standard deviation and median. The significance of the obtained results was judged at the $5 \%$ level.

\subsection{Tests Were Used}

Chi-square test $\left(\chi^{2}\right)$, Fisher's Exact or Monte Carlo correction $\left({ }^{\mathrm{MC}} \mathrm{p},{ }_{\mathrm{FE}} \mathrm{p}\right.$ ), Paired t-test, Wilcoxon signed ranks test, Regression and Kaplan-Meier.

\section{Results}

In our study, there was no statistical difference between the 2 groups in terms of demographic and clinical data as regards age, sex, viral markers, presence of signs of portal hypertension, preoperative serum albumin, preoperative serum bilirubin, preoperative AFP (Table 1).

Table 1. Preoperative demographics and work-up.

\begin{tabular}{llll}
\hline variable & Group A (24) & Group B (15) & P value \\
\hline Age (years) & $59.05 \pm 6.26$ & $60.43 \pm 7.91$ & 0.569 \\
Sex (M/F) & $23 / 1$ & $13 / 2$ & 0.551 \\
HCV (yes/ttt) & $19 / 5$ & $13 / 2$ & 1.000 \\
AFP level (ng/ml) & $215.3 \pm 652.3$ & $207.9 \pm 326.6$ & 0.210 \\
Esophageal varices (yes/no) & $9 / 15$ & $8 / 7$ & 0.678 \\
Spleen size (cm) & $14.73 \pm 2.73$ & $14.50 \pm 1.92$ & 0.792 \\
Preoperative Plt & $146.3 \pm 66.58$ & $189.3 \pm 91.03$ & 0.096 \\
Preoperative albumin (gm/dl) & $3.88 \pm 0.38$ & $3.98 \pm 0.47$ & 0.484 \\
Preoperative bilirubin (mg/dl) & $0.84 \pm 0.41$ & $0.76 \pm 0.40$ & 0.454 \\
\hline
\end{tabular}

As regards to the size of the HCC lesion (Table 2), group A ranged between 2 and $5 \mathrm{~cm}$ while group B included sizes ranged between 5.5 and $16 \mathrm{~cm}$. 
Table 2. Tumor characteristics and surgical types.

\begin{tabular}{llll}
\hline variable & Group A (24) & Group B (15) & P value \\
\hline Tumor size (mean) & $4.12 \pm 0.85$ & $9.57 \pm 3.40$ & $0.006^{*}$ \\
Operative time (min) & $167.4 \pm 42.36$ & $215.0 \pm 54.31$ & $<0.001^{*}$ \\
Intra operative blood loss (cc) & $319.1 \pm 226.1$ & $657.1 \pm 361.5$ & 0.139 \\
ICU stay (days): & $1.62 \pm 0.87$ & $2.38 \pm 1.39$ & 0.052 \\
Hospital stay (days): & $6.24 \pm 3.82$ & $9.36 \pm 6.92$ & \\
Resection type (no): & & 3 & \\
Non-anatomical. & 20 & 6 & \\
RT. hepatectomy. & 1 & 3 & \\
LT. hepatectomy. & 0 & 2 & \\
LT. lateral hepatectomy. & 3 & 8 & \\
Pathology grading: & & 5 \\
Grade 1: & 9 & $6 / 9$ \\
Grade 2: & 10 & 1 \\
Grade 3: & 5 & \\
Micro vascular invasion (yes/ no): & $7 / 17$ & & \\
Resection margin (positive): & 1 & & \\
\hline
\end{tabular}

All patients in both groups underwent open hepatectomy (Table 2), patterns of resection involved non-anatomical resection, Rt. hepatectomy, Lt. hepatectomy and left lateral hepatectomy. There was no ultra-major liver resection (extended right and left hepatectomy).

In group $\mathrm{A}$, the mean operative time was $167.4 \pm 42.36$ minutes and in group B was 215.0 \pm 54.31 minutes (Table 2), which was statistically significant (P- 0.006). The mean intraoperative blood loss in group A was of $319.1 \pm 226.1 \mathrm{cc}$ and in group B was $657.1 \pm 361.5 \mathrm{cc}$ which was statistically significant $(p<0.001)$ (Table 2), The main causes of intraoperative bleeding include bleeding that happened during parenchymal transection or liver mobilization and injuries of the short hepatic veins. Bleeding was controlled by the usual hemostatic measures using either metal clips, Figure of 8 sutures and electrocautery devices. This bleeding was controllable and passed without any significant complications.
Collectively 29 patients (74.3\%) were admitted to ICU. There was no statistically significant difference, as was also for the hospital stay.

As regards histopathological examination of the specimens, in group A, adenocarcinoma grade 1 in 9 patients $(37.5 \%)$ and grade 2 in 10 patients $(41.6 \%)$ and grade 3 in 5 patients $(20.9 \%)$ while in group $B$, grade 1 in 2 patients (14.3\%), grade 2 in 8 patients $(52.4 \%)$ and grade 3 in 5 patients $(33.3 \%)$. There was no statistically significant difference in both groups regarding histological grade ( $\mathrm{P}$ value 0.335$)$. In group $A$, microvascular invasion in 7 patients $(28.6 \%)$ while in group B, in 6 patients $(40 \%)$ with no statistical significance (P- value 0.477). Regarding resection margin in groups $\mathrm{A}$ and $\mathrm{B}$ it was positive in only 1 patient in each group $(5.7 \%$ and $7.1 \%)$ respectively, with no statistical significance (P-value 1).

Table 3. Intra and post-operative complications.

\begin{tabular}{|c|c|c|c|c|c|c|c|c|}
\hline \multirow{2}{*}{ Complications } & \multicolumn{2}{|c|}{ Total $(n=39)$} & \multicolumn{2}{|c|}{ Group $A \leq 5(n=24)$} & \multicolumn{2}{|c|}{ Group B $>5(n=15)$} & \multirow{2}{*}{$\chi^{2}$} & \multirow{2}{*}{${ }^{\mathrm{MC}} \mathbf{p}$} \\
\hline & No. & $\%$ & No. & $\%$ & No. & $\%$ & & \\
\hline \multicolumn{9}{|l|}{ Intra-operative } \\
\hline No & 31 & 80.0 & 22 & 90.5 & 9 & 64.3 & \multirow{4}{*}{5.796} & \multirow{4}{*}{0.052} \\
\hline Bleeding & 6 & 14.3 & 1 & 4.8 & 5 & 33.3 & & \\
\hline LHD injured & 1 & 2.9 & 1 & 4.8 & 0 & 0.0 & & \\
\hline Diaphragmatic tear & 1 & 2.9 & 0 & 0.0 & 1 & 7.1 & & \\
\hline No & 14 & 37.1 & 11 & 47.6 & 3 & 21.4 & \multirow{10}{*}{11.830} & \multirow{10}{*}{0.125} \\
\hline Liver decompensation & 2 & 5.7 & 1 & 4.8 & 1 & 7.1 & & \\
\hline Chest infection & 5 & 14.3 & 3 & 14.3 & 2 & 14.3 & & \\
\hline pleural effusion & 2 & 5.7 & 2 & 9.5 & 0 & 0.0 & & \\
\hline Wound seroma & 2 & 5.7 & 0 & 0.0 & 2 & 14.3 & & \\
\hline Ascites & 5 & 14.3 & 3 & 14.3 & 2 & 14.3 & & \\
\hline Ascites\& pleural effusion & 2 & 5.7 & 0 & 0.0 & 2 & 14.3 & & \\
\hline Wound infection\& collection & 1 & 2.9 & 0 & 0.0 & 1 & 7.1 & & \\
\hline Bile leak \& collection & 2 & 5.7 & 1 & 4.8 & 1 & 7.1 & & \\
\hline Wound infection & 3 & 8.6 & 3 & 14.3 & 0 & 0.0 & & \\
\hline
\end{tabular}


Intraoperative complications included bleeding and diaphragmatic injury. Postoperative complications included chest infection, pleural effusion, ascitis bile leak that was managed non-operatively by ERCPand CBD stenting and collection and wound infection. (Table 3 ).

We had 2 peri-operative mortalities, both patients died due to post-hepatectomy liver cell failure, they failed to regain their liver functions early. One belonged to group A and the other one belonged to group B. Two patients dropped out from the follow-up program, one from group A after 2 months and the other one from group B after 4 months.

Table 4. Investigations after 1 month.

\begin{tabular}{|c|c|c|c|c|c|}
\hline After 1 month & Total $(n=37)$ & Group $A \leq 5(n=23)$ & Group B $>5(n=14)$ & Test of sig. & p \\
\hline Albumin & & & & & \\
\hline $\begin{array}{l}\text { Mean } \pm \text { SD. } \\
\text { Bilirubin }\end{array}$ & $3.66 \pm 0.32$ & $3.66 \pm 0.33$ & $3.66 \pm 0.31$ & & \\
\hline $\begin{array}{l}\text { Mean } \pm \text { SD. } \\
\text { Platelets }\end{array}$ & $1.12 \pm 0.30$ & $1.10 \pm 0.27$ & $1.16 \pm 0.34$ & & \\
\hline $\begin{array}{l}\text { Mean } \pm \text { SD. } \\
\text { INR }\end{array}$ & $112.8 \pm 23.24$ & $113.5 \pm 20.09$ & $111.7 \pm 28.09$ & $\mathrm{U}=125.0$ & 0.474 \\
\hline Mean \pm SD. & $1.10 \pm 0.09$ & $1.10 \pm 0.08$ & $1.09 \pm 0.10$ & & \\
\hline
\end{tabular}

One month after surgery serum albumin level, serum total bilirubin, INR showed no statistically significant difference between patients of group A and B, (3.66 \pm 0.33 and 3.66 \pm 0.31$)(1.10 \pm 0.27$ and $1.16 \pm 0.34)(1.10 \pm 0.27$ and $1.09 \pm 0.10)$ respectively (Table 4).

Table 5. Recurrence and its management.

\begin{tabular}{|c|c|c|c|c|c|c|c|c|}
\hline & \multicolumn{2}{|c|}{ Total $(n=35)$} & \multicolumn{2}{|c|}{ Group $A \leq 5(n=22)$} & \multicolumn{2}{|c|}{ Group B >5 $(n=13)$} & \multirow{2}{*}{$\chi^{2}$} & \multirow{2}{*}{$\mathbf{p}$} \\
\hline & No. & $\%$ & No. & $\%$ & No. & $\%$ & & \\
\hline \multicolumn{9}{|l|}{ Recurrence } \\
\hline No & 23 & 65.7 & 15 & 66.7 & 8 & 64.3 & \multirow{4}{*}{0.021} & \multirow{4}{*}{${ }^{\mathrm{FE}} \mathrm{p}=1.000$} \\
\hline Yes & 12 & 34.3 & 7 & 33.3 & 5 & 35.7 & & \\
\hline Time of recurrence (months) & \multicolumn{2}{|l|}{$(n=12)$} & \multicolumn{2}{|l|}{$(n=7)$} & \multicolumn{2}{|l|}{$(n=5)$} & & \\
\hline Min. - Max. & \multicolumn{2}{|l|}{$9.0-22.0$} & \multicolumn{2}{|l|}{$10.0-22.0$} & \multicolumn{2}{|l|}{$9.0-20.0$} & & \\
\hline Mean \pm SD. & \multicolumn{2}{|l|}{$15.50 \pm 4.23$} & \multicolumn{2}{|l|}{$15.86 \pm 4.49$} & \multicolumn{2}{|l|}{$15.0 \pm 4.30$} & \multirow[t]{2}{*}{$\mathrm{t}=0.332$} & 0.747 \\
\hline Site of recurrence & \multicolumn{2}{|l|}{15.50} & \multicolumn{2}{|l|}{16.0} & \multicolumn{2}{|l|}{15.0} & & \\
\hline No & 23 & 65.7 & 14 & 66.7 & 9 & 64.3 & \multirow{4}{*}{1.505} & \multirow{4}{*}{${ }^{\mathrm{MC}} \mathrm{p}=0.673$} \\
\hline Intrahepatic & 11 & 31.4 & 7 & 33.3 & 4 & 28.6 & & \\
\hline Lung & 1 & 2.9 & 0 & 0.0 & 1 & 7.1 & & \\
\hline \multicolumn{7}{|l|}{ TTT of recurrence } & & \\
\hline No & 23 & 65.7 & 14 & 66.7 & 9 & 64.3 & \multirow{2}{*}{1.421} & \multirow{2}{*}{${ }^{\mathrm{MC}} \mathrm{p}=0.830$} \\
\hline TACE & 4 & 11.4 & 3 & 14.3 & 1 & 7.1 & & \\
\hline
\end{tabular}

All patients in both groups were followed-up postoperatively with serum AFP and abdominal U/S for early detection of HCC recurrence. In suspected cases for recurrence, triphasic $\mathrm{CT}$ and/or MRI were done.

In group $\mathrm{A}, \mathrm{HCC}$ has recurred in 7 patients $(33.3 \%)$, mean time of recurrence was $15.86 \pm 4.49$ ranged between $10-22$ months. De novo lesions (intrahepatic recurrence) has occurred in a remote pattern away from the site of resection in all the patients $(100 \%)$. These patients with recurrent HCC were managed as follows, radiofrequency ablation (RFA) in 3 patients, trans-arterial chemoembolization (TACE) in another 3 patients and best supportive care (BSC) in 1 patient. In group B, 5 patients $(35.7 \%)$ developed recurrent $\mathrm{HCC}$ and the time of recurrence ranged between 9-20 months with a mean time of $15.0 \pm 4.30$. Remote liver recurrence occurred in 4 patients $(28.6 \%)$ while metastasis at the lung occurred in 1 patient $(7.1 \%)$. Recurrent $\mathrm{HCC}$ in these patients were managed: 2 patients (14.3\%) were managed by RFA, 1 patient $(7.1 \%)$ was managed by TACE, 1 patient $(7.1 \%)$ had an advanced disease which was beyond any intervention and managed by BSC and the patient with extrahepatic recurrence $(7.1 \%)$ was beyond any intervention and managed by BSC. There was no statistically significant difference between the two studied groups regarding recurrence.

Table 6. Overall and disease free survival.

\begin{tabular}{|c|c|c|c|c|c|c|c|c|}
\hline & \multicolumn{2}{|c|}{ Total $(n=35)$} & \multicolumn{2}{|c|}{ Group $A \leq 5(n=22)$} & \multicolumn{2}{|c|}{ Group $B>5(n=13)$} & \multirow{2}{*}{$\chi^{2}$} & \multirow{2}{*}{${ }^{\mathrm{FE}} \mathbf{p}$} \\
\hline & \begin{tabular}{|l} 
No. \\
\end{tabular} & $\%$ & \begin{tabular}{|l|} 
No. \\
\end{tabular} & $\%$ & \begin{tabular}{|l|} 
No. \\
\end{tabular} & $\%$ & & \\
\hline Disease free survival (2 ye & & & & & & & \multirow{3}{*}{0.021} & \multirow{3}{*}{1.000} \\
\hline No & 12 & 34.3 & 7 & 33.3 & 5 & 35.7 & & \\
\hline $\begin{array}{l}\text { Yes } \\
\text { Overall survival ( } 2 \text { years })\end{array}$ & 23 & 65.7 & 15 & 66.7 & 8 & 64.3 & & \\
\hline
\end{tabular}




\begin{tabular}{|c|c|c|c|c|c|c|c|c|}
\hline & \multicolumn{2}{|c|}{ Total $(n=35)$} & \multicolumn{2}{|c|}{ Group $A \leq 5(n=22)$} & \multicolumn{2}{|c|}{ Group B >5 (n=13) } & \multirow{2}{*}{$\chi^{2}$} & \multirow{2}{*}{${ }^{\mathrm{FE}} \mathbf{p}$} \\
\hline & No. & $\%$ & No. & $\%$ & No. & $\%$ & & \\
\hline $\begin{array}{l}\text { Alive } \\
\text { Died }\end{array}$ & $\begin{array}{l}26 \\
9\end{array}$ & $\begin{array}{l}74.3 \\
25.7\end{array}$ & $\begin{array}{l}17 \\
5\end{array}$ & $\begin{array}{l}76.2 \\
23.8\end{array}$ & $\begin{array}{l}9 \\
4\end{array}$ & $\begin{array}{l}71.4 \\
28.6\end{array}$ & 0.100 & 1.000 \\
\hline
\end{tabular}

Twenty-six patients $(74.3 \%)$ were alive till the end of the follow-up period of the research study. In group A, 76.2\% (17 patients) while in group B, 71.4\% (9 patients), was no statistically significant difference (P- value 1). As Regards disease-free survival (DFS), 23 patients $(65.7 \%)$ till the end of the follow-up period of the research study were surviving without recurrence. In group A, DFS was $66.7 \%$ (15 patients) and in group B, DFS was $64.3 \%$ (8 patients). Also, there was no statistically significant significance (Table 6).

Table 7. Univariate and multivariate analysis of factors affecting recurrence.

\begin{tabular}{lllll}
\hline & Univariate & & ${ }^{*}$ Multivariate \\
\cline { 2 - 5 } & $\mathbf{p}$ & OR (95\%C. I) & p & OR (95\%C. I) \\
\hline Tumor size & 0.885 & $1.015(0.830-1.241)$ & - & - \\
Path Differentiation (poor differentiation) & $0.025^{*}$ & $6.667(1.269-35.035)$ & 0.359 & $3.013(0.285-31.875)$ \\
Path Microvascular invasion & $<0.001^{*}$ & $52.50(6.431-428.574)$ & $0.001^{*}$ & $41.242(4.871-349.165)$ \\
\hline
\end{tabular}

In a univariate and multivariate analysis of the factors affecting recurrence, the size of the tumor wasn't found to be an independent factor affecting recurrence on the other hand pathological differentiation; mainly the poorly differentiated tumors and microvascular invasion were found to be an independent factor affecting recurrence (Table 7).

Table 8. Univariate and multivariate analysis of factors affecting overall survival.

\begin{tabular}{lllll}
\hline & Univariate & & Multivariate \\
\cline { 2 - 5 } & $\mathbf{p}$ & OR (95\%C. I) & p & OR (95\%C. I) \\
\hline Tumor size & 0.670 & $1.047(0.847-1.294)$ & - & - \\
Path Differentiation & $0.026^{*}$ & $6.875(1.266-37.342)$ & 0.070 & $5.353(0.871-32.896)$ \\
Path Microvascular invasion & 0.128 & $3.393(0.703-16.385)$ & 0.481 & $1.900(0.319-11.306)$ \\
\hline
\end{tabular}

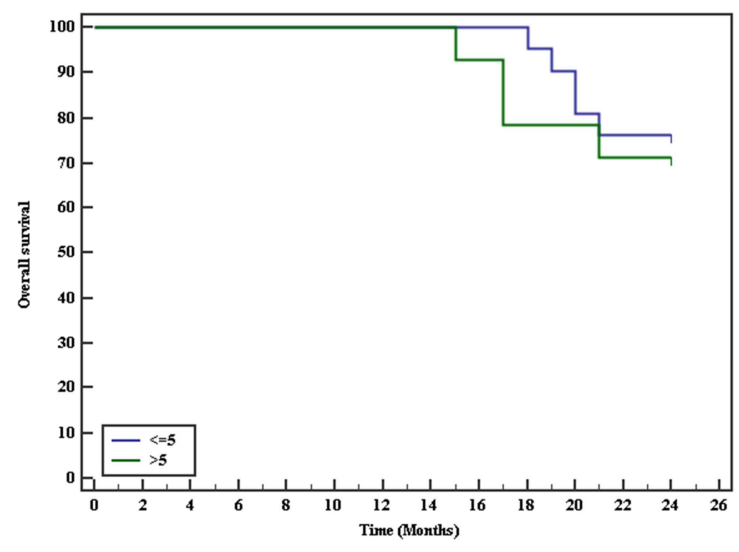

Figure 8. Kaplan-Meier survival curve for overall survival between both groups according to tumor size.

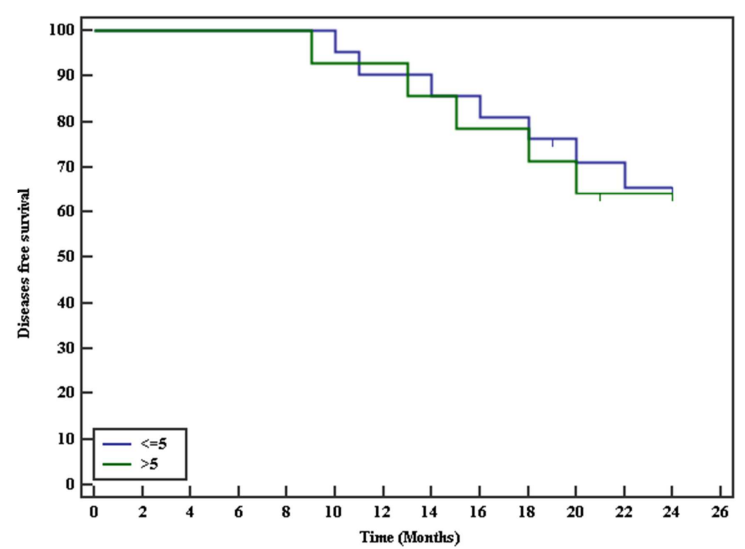

Figure 9. Kaplan-Meier survival curve for disease free survival between both groups according to tumor size.

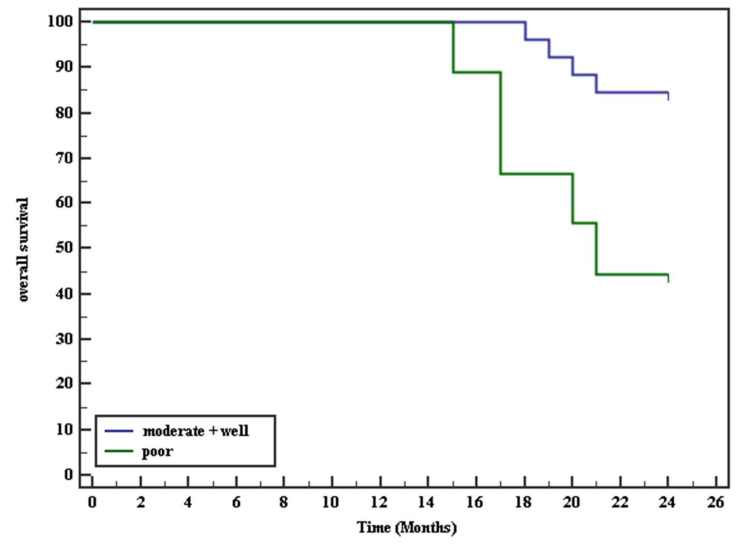

Figure 10. Kaplan-Meier survival curve for overall survival between both groups regarding pathological differentiation.

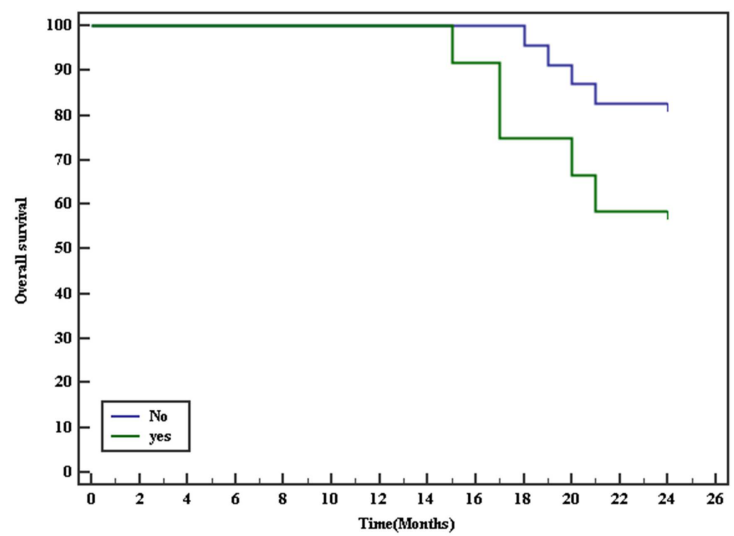

Figure 11. Kaplan-Meier survival curve for overall survival according to pathological microvascular invasion. 
In a univariate and multivariate analysis of the factors affecting overall survival, pathological differentiation was found to be independent factor affecting overall survival but tumor size and micro invasion weren't found to be independent factors (Table 8, Figures 8-11).

\section{Discussion}

In patients with large-sized HCC lesions, it was traditionally thought that their long-term prognosis is considered to be poor. This may be related with a higher incidence of satellite nodules, occult vascular invasion, more aggressive tumor biology, the carcinogenic potential of the non-tumorous cirrhotic liver, the propensity of $\mathrm{HCC}$ to spread along intra-segmental portal vein branches and more advanced histologic grade in large HCC than small HCC. [2]

In previous studies, the size of HCC lesion has been considered an independent risk factor for patient survival and tumor recurrence, but this concept has been modified after multi-center research showed that survival outcomes were independent of tumor size in patients underwent resection of solitary HCC without vascular invasion. [10]

The cutoff value that has an influence on the survival after resection for $\mathrm{HCC}$ was defined as $5 \mathrm{~cm}$ in the 7 th edition of AJCC cancer staging system. [11]

All HCC patients in our study were associated with cirrhosis, they all were CTP class A, child B and C patients weren't offered liver resection because of the high morbidity and mortality associated with these classes and other treatment options were offered by the HCC tumor board according to the guidelines. Our results matched with the results of a study on liver resection for large tumors more than $5 \mathrm{~cm}$ by Zhao et al 2016 in which all patients were cirrhotic and were Child class A.

Evaluating the degree of fibrosis by liver stiffness measurement with transient elastography is crucial. A recent study demonstrated that the degree of fibrosis affecting the underlying parenchyma had no impact on postoperative outcomes following the resection of large HCC $(\geq 5 \mathrm{~cm})$. In such tumors, prognosis appeared to be correlated to pathological features (microvascular invasion, satellite nodules, the grade of differentiation and resection margins) rather than to the underlying parenchyma. In the event of fibrosis $\leq \mathrm{F} 3$, hepatic resection remains the gold standard for curative treatment. [13]

A FLR (future liver remnant) of $40-50 \%$ of the TLV (total liver volume) should be preserved after major LR in patients with cirrhosis [14]. In our study, $>40-50 \%$ FLR has been used as a safety cut-off point and patients with tumors $>5 \mathrm{~cm}$ who were booked for major hepatic resection a CT volumetric study was done making sure of the adequate FLR $>40-50 \%$.

In our study 7 patients had platelets count below 100,000, 3 patients had minor resections and 4 patients had a major liver resection and passed without major complications. For patients undergoing liver resection for $\mathrm{HCC}$, portal hypertension (PHT) is associated with worse liver fibrosis, impaired postoperative course including higher rates of postoperative liver dysfunction, ascites and increased mortality as well as longer hospital stays. [14]

In our study group A patients had less operative time (mean 167.4 $\pm 42.36 \mathrm{~min}$ ) compared to group B patients (mean $215.0 \pm 54.31 \mathrm{~min}$ ), this was of statistically significant importance (P-value 0.006). In a study by Tiffany et al 2014 they had a significantly longer operative time for large tumors than smaller ones but they didn't show risk difference in complications including postoperative liver failure between patients had major and minor resections.

In our study for group A patients intraoperative blood loss ranged between 150 and $1100 \mathrm{cc}$ with a mean of $319.1 \pm 226.1$ cc, and for group B patients intraoperative blood loss ranged between 250 and $1300 \mathrm{cc}$ with a mean of $657.1 \pm 361.5 \mathrm{cc}$, which showed statistically significant difference (P-value $<$ 0.001 ) between the 2 studied groups suggesting that with increased tumor size there is a higher chance for intraoperative bleeding.

In our study for group A patients, intraoperative blood transfusion ranged between $500-1500 \mathrm{ml}$ with a mean of $833.33 \pm 408.25$, while in group B patients' blood transfusion ranged between $500-2000 \mathrm{ml}$ with mean $1166.67 \pm 559.02$, with no statistically significant difference between both groups. This was matching with a recent study by Hokuto et al 2018 who demonstrated that the estimated amount of intraoperative blood loss, frequency of transfusions, and postoperative morbidity rates were similar in both groups of patients who had anatomical Vs non-anatomical resection for small and large HCC tumors.

In our study, in group A patients the overall survival was $76.2 \%$ and disease-free survival was $66.7 \%$ while in group B patients the overall survival $71.4 \%$ and disease-free survival was $64.3 \%$ with no statistically significant difference. This indicates that the size alone is not an independent factor for recurrence and DFS.

The importance of tumor size as a prognostic factor for HCC remains controversial today. Although traditionally, size has long been considered an important prognostic factor for HCC subsequent large cohort studies demonstrated that the negative correlation between size and survival rate was largely due to the association of tumor size with other more important adverse prognostic factors such as vascular invasion, poorer differentiation, and multifocality.

Several large studies had also subsequently, down-played the significance of tumor size by demonstrating its lack of significance as a prognostic factor for solitary HCC. [17] However, more recently, several large cohort studies have demonstrated the importance of tumor size as a prognostic marker for solitary HCC and challenged the rationale for its omission as an important prognostic factor. [10]

In a study by Goh et al 2017, they documented that tumor size was an independent prognostic factor of RFS after LR for solitary HCC supporting the observations of recent large single-center studies that tumor size is an important prognostic factor of surgically-resected solitary HCC. [20]

Review articles analyzed the results of resection for huge 
HCCs $>10 \mathrm{~cm}$, in which the perioperative mortality rates were approximately 3\% (range 1.2-6.9\%); 5-year overall patient survival rates were approximately $30 \%$ (range 19-39\%); and 1 -year recurrence rates were approximately $60 \%$ (range $57-$ $72 \%$ ). The common risk factors for patient survival were a vascular invasion, multiple lesions, and high AFP. [20]

It is generally accepted that there is no size limit that precludes hepatic resection, especially for solitary huge HCCs if these tumors are respectable. There are two aspects to the operability of huge HCCs, namely, operative safety and surgical curability. Resection of large HCC is often associated with a high risk of bleeding, as well as being a demanding surgical procedure; thus patients with preserved liver function are usually selected. Another drawback to surgery is the presence of intrahepatic metastases in the future remnant liver, beyond the permissible extents of hepatic resection. [20]

\section{Conclusion}

Our results suggest that tumor size alone doesn't affect the safety and efficacy of surgical resection for large HCC and that surgery should be considered in the treatment for large HCC safely in selected patients. Our study showed that hepatectomy for large HCC could be performed with an acceptable morbidity and mortality rate. With the improvement in patient selection and treatment strategy, solitary large HCC is not a contraindication to surgical therapy. The small sample size limits the results so more studies with large sample sizes are required to properly assess these data.

\section{References}

[1] Lafaro, K. J., Deemirjian, A. N., Pawlik, T. M. (2015). Epidemiology of Hepatocellular Carcinoma. Surg Oncol Clin N Am, 24: 1-17.

[2] Garancini, M., Pinotti, E., Nespoli, S., Romano, F. (2016). Hepatic Resection Beyond Barcelona Clinic Liver Cancer Indication: When and How. World Journal of Hepatology, 8 (11): 513-516.

[3] Aziz, A. M., Zakaria, H., Ayuob, I., Soliman, H. E., and Othman, M. (2016). The safety and adequacy of liver resection for large hepatocellular carcinoma: A retrospective single institute study. Saudi surgical journal, 4: 20-28.

[4] Wong, T. L., Cheung, T. T., Chok, K. S., Poon, R. T. (2014). Treatment Strategy to Improve Long Term Survival for Hepatocellular Carcinoma Smaller than $5 \mathrm{~cm}$ : Major Hepatectomy Vs Minor Hepatectomy. World Journal of Surgery, 38: 2386-2394.

[5] Lim, K. C., Chow, P. K., Allen, J. C., Siddiqui, F. J. (2012). Systemic Review of Outcomes of Liver Resection for Early Hepatocellular Within Milan Criteria. British Journal of Surgery, 99 (12): 1622-1629.

[6] Guglielmi, A., Ruzzenente, A., Conci, S., Valdegamberi, A. (2014). Hepatocellular Carcinoma: Surgical Perspectives Beyond the Barcelona Clinic Liver Cancer Recommendations.
World Journal of Gastroenterology, 20: 7527-7533.

[7] Chan, Y. C., Kabiling, C. S., Pillai, V. G., Gusatavo, A. (2015). Survial Outcomes Between Hepatic Resection and Transarterial Embolization for Hepatocellular Carcinoma More than $10 \mathrm{~cm}$ : A Propensity Score Model. World Journal of Surgery, 39: 1510-1518.

[8] Lim, C., Sakamoto, Y., Yamamoto, S., Kokudo, N., Makuuchi, M. (2014). Above $5 \mathrm{~cm}$, Size Doesn't Matter Anymore in Patients with Hepatocellular Carcinoma. World Journal of Surgery, 38: 2910-2918.

[9] Poon, D., Anderson, B. O., Chen, L. T., Tanaka, K. (2009). Asian Oncology Summit Management of Hepatocellular Carcinoma in Asia. Lancet oncololgy, 10 (11): 1111-1118.

[10] Hwang, J., Kim, S. H., Lee, M. W. and Lee, J. Y. (2012) Small $(<2 \mathrm{~cm})$ hepatocellular carcinoma in patients with chronic liver disease: a comparison of gadoxetic acidenhanced 3. $0 \mathrm{~T}$ MRI and multiphasic 64-multiraw detector CT. British Journal of Radiololgy, 85 (1-15): 314-322.

[11] Noh, J. H., Kim, T. S., Ahn, K. S., Kim, Y. H. and Kang, K. J. (2016). Prognostic factors after hepatic resection for the single hepatocellular carcinoma larger than $5 \mathrm{~cm}$. Annals of Surgical Treatment and Research, 91 (3): 104-111.

[12] Zhao, H. C., Wu, R. L., Liu. F. B., Zhao, Y. J. (2016). A Retrospective Analysis of Long Term Outcomes in Patients Undergoing Hepatic Resection for Large $(>5 \mathrm{~cm})$ Hepatocellular Carcinoma. Hepatopancreatobiliary: 1-7.

[13] Golsea, N., El Bouyousfib, A., Marques, F., Bancel, B., Mohkamb, K., Ducerf, C., Merle, P., Sebaghe, M., and Castaing, D. (2018). Large hepatocellular carcinoma: Does fibrosis really impact prognosis after resection? Journal of Visceral Surgery, 155: 265-273.

[14] Cauchy, F., Soubrane, O., Belghiti, J. (2014). Liver resection for HCC: Patient's selection and controversial scenarios. Best Practice \& Research Clinical Gastroenterology, 28: 881-889.

[15] Tiffany, C. L., Tan, T. C., Kenneth, S. H., Albert, C. Y., Poon, R. T. (2014). Treatment Strategy to Improve Long-Term Survival for Hepatocellular Carcinoma Smaller than $5 \mathrm{~cm}$ : Major Hepatectomy vs Minor Hepatectomy. World Journal of Surgery, 38: 2386-2394.

[16] Hokuto, D., Nomi, T., Yasuda, S., Yoshikawa, T., Yamada, T., (2018). Does anatomic resection improve the postoperative outcomes of solitary hepatocellular carcinomas located on the liver surface? Surgery, 163: 285-90.

[17] Kluger, M. D., Salceda, J. A., Laurent, A., Tayar, C., Decaens, T., Cherqui, D. (2015). Liver resection for hepatocellular carcinoma in 313 Western patients: Tumor biology and underlying liver rather than tumor size drive prognosis. Journal of Hepatology, 62: 1131-1140.

[18] Hwang, S., Lee, Y. J., Kim, K. H., Ahn, C. S., Moon, D. B., (2015). Long-Term Outcome after Resection of Huge Hepatocellular Carcinoma $>10 \quad \mathrm{~cm}$ : Single-Institution Experience with 471 Patients. World Journal of Surgery, 39: 2519-2528.

[19] Goh, B. K., Teo, J. Y., Chan, C. Y., Lee, S. Y., Jeyaraj, P., Cheow, P. C., (2016). Importance of Tumor Size as a Prognostic Factor after Partial Liver Resection for Solitary Hepatocellular Carcinoma: Implications on the Current AJCC Staging System. Journal of Surgical Oncology, 113: 89-93. 
[20] Hwang, S., Lee, Y. J., Kim, K. H. (2015). The impact of tumor size on long-term survival outcomes after resection of solitary hepatocellular carcinoma: Single-institution experience with 2,558 patients. Journal of Gastrointestinal Surgery, 19: 12811290. 